\title{
Solid Papillary Carcinoma of Breast: Clinicopathologic Comparison With Conventional Ductal Carcinoma of Breast
}

\author{
Atif A. Hashmi ${ }^{1}$, Syeda N. Iftikhar ${ }^{1}$, Rimsha Haider ${ }^{2,3}$, Raviha Haider ${ }^{4}$, Muhammad Irfan ${ }^{5}$, Javaria Ali ${ }^{1}$ \\ 1. Pathology, Liaquat National Hospital and Medical College, Karachi, PAK 2. Internal Medicine, Liaquat National \\ Hospital and Medical College, Karachi, PAK 3. Emergency Medicine, National Institute of Blood Diseases and Bone \\ Marrow Transplantation, Karachi, PAK 4. Internal Medicine, Ziauddin Medical University, Karachi, PAK 5. Statistics, \\ Liaquat National Hospital and Medical College, Karachi, PAK
}

Corresponding author: Atif A. Hashmi, atifhashmi345@gmail.com

\section{Abstract \\ Introduction}

Solid papillary carcinoma (SPC) is a distinct rare subtype of breast tumour that often exhibits a neuroendocrine differentiation. Due to the rarity of these tumours, few studies have assessed the clinicopathological features of these tumours. Therefore, in this study, we evaluated the clinical and pathological profiles of SPC and compared the pathologic features with conventional invasive ductal carcinoma (IDC) in our population.

\section{Methods}

It was a retrospective cross-sectional study conducted at Liaquat National Hospital and Medical College from January 2013 until December 2019 over seven years. Cases with histological diagnosis of SPC and IDC were included in the study, and clinicopathological characteristics were compared.

\section{Results}

We included 39 cases of SPC in our study diagnosed during the study period. During the same timeline, 634 cases of IDC were reported and therefore included in the study for comparison. The mean age of the patients with SPC was $53.97 \pm 12.15$ years, and the mean tumour size was $3.42 \pm 1.87 \mathrm{~cm}$. Axillary metastasis was noted in $15.4 \%$ of cases. $94.9 \%$ of cases of SPC were invasive. Estrogen receptor (ER), progesterone receptor (PR), human epidermal growth factor receptor-2 (HER2/neu) and synaptophysin positivity was seen in $84.6 \%, 87.2 \%, 10.3 \%$, and $59 \%$ respectively. Recurrence was noted in $10.3 \%$ of cases with $94.9 \%$ survival rate. Cases of SPC had significantly lower grade (grade I + II), tumour (T) and nodal (N) stage than IDC. Moreover, the frequency of hormonal receptor expression (ER and PR) was higher, and the frequency of human epidermal growth factor receptor 2 (HER2/neu) expression was lower compared to IDC.

Review began 10/17/2020 Review ended 10/26/2020 Published 10/26/2020

๑) Copyright 2020

Hashmi et al. This is an open access article distributed under the terms of the Creative Commons Attribution License CC-BY 4.0., which permits unrestricted use, distribution, and reproduction in any medium, provided the original author and source are credited.

\section{Conclusion}

SPC is a distinct variant of malignant papillary breast tumours with overall better prognostic parameters than IDC. Therefore, it is essential to recognize the histological features of this rare breast tumour.

Categories: Pathology, General Surgery, Oncology

Keywords: solid papillary carcinoma, papillary breast tumors, breast cancer, invasive ductal carcinoma, synaptophysin, estrogen receptor, progesterone receptor, human epidermal growth factor receptor-2

\section{Introduction}

In Southeast Asia, breast cancers are common and typically present late and are associated with adverse prognostic parameters [1,2]. Invasive ductal carcinoma (IDC) of the breast is the most common histologic subtype of breast cancer with a distinct pathological and prognostic profile $[3,4]$. Papillary breast neoplasms are a heterogeneous group of tumours, ranging from benign intraductal papilloma to malignant invasive papillary carcinoma [5]. Between these two extremes, there lie categories of in situ papillary carcinoma. Two notoriously deceptive in situ tumours in this category include solid papillary carcinoma (SPC) and encapsulated papillary carcinoma. SPC is further divided into SPC in situ and SPC invasive based on the presence or absence of invasive component. Although SPC in situ mostly lacks a myoepithelial layer at the periphery of the tumour, they are still considered in situ tumours. Histologically, SPC is characterized by multiple circumscribed nodules of a tumour with a smooth outline [6]. They often show neuroendocrine differentiation, characterized by synaptophysin positivity and sometimes also exhibit mucinous component. The invasive component in SPC can be in the form of conventional invasive ductal carcinoma (IDC) or invasive carcinoma with SPC like features. The invasive component derives the tumour stage (T-stage). Due to the rarity of these tumours, few studies have assessed the clinicopathological features of these tumours. 


\section{Cureus}

Therefore, in this study, we evaluated the clinical and pathological profiles of SPC and compared the pathologic features with conventional IDC in our population.

\section{Materials And Methods}

It was a retrospective cross-sectional study conducted at Liaquat National Hospital and Medical College from January 2013 until December 2019 over seven years. All patients underwent resections of breast tumours after biopsy diagnosis of atypical papillary breast tumours. Specimens included a lumpectomy, simple mastectomy with sentinel lymph node dissection and modified radical mastectomy (MRM). Intraoperative frozen section for sentinel lymph nodes was performed for patients with clinically and radiologically negative axillary lymph nodes. In cases where the operative plan was mastectomy, any positive sentinel lymph node (macrometastasis) on the frozen section was followed by axillary lymph node dissection. Alternatively, for patients undergoing breast conservation surgery, a minimum of three positive sentinel lymph nodes on the frozen section with at least one macrometastasis was followed by axillary dissection. The axillary dissection without prior sentinel lymph node biopsy was performed in cases with clinically or radiologically positive lymph nodes after trucut biopsy/fine needle aspiration cytology confirmation of malignancy. Gross examination and sampling of tumours were done according to standard protocols. Hematoxylin and eosin-stained sections were first examined, and then myoepithelial stains were applied on representative tissue blocks. Cases with histological diagnosis of SPC and IDC were included in the study, and clinicopathological characteristics were compared. Estrogen receptor (ER), progesterone receptor (PR) and human epidermal growth factor receptor-2 (HER2/neu) immunohistochemistry (IHC) was applied to all cases of SPC and IDC. Cases with equivocal (2+) IHC for HER2/neu were further tested by fluorescence in situ hybridization (FISH). Cases with SPC, there were two cases with equivocal HER2/neu IHC (2+), and both were FISH negative. For IDC, 64 cases were equivocal (2+) for HER2/neu IHC, out of which 38 cases revealed HER2/neu amplification on FISH and therefore labelled as Her2/neu positive on the final analysis. The rest of the cases (non-amplified) were taken as HER2/neu negative. Also, synaptophysin IHC was performed on all cases of SPC. Histological images and myoepithelial stains of in situ and invasive SPC are shown in Figures 1, 2, respectively.

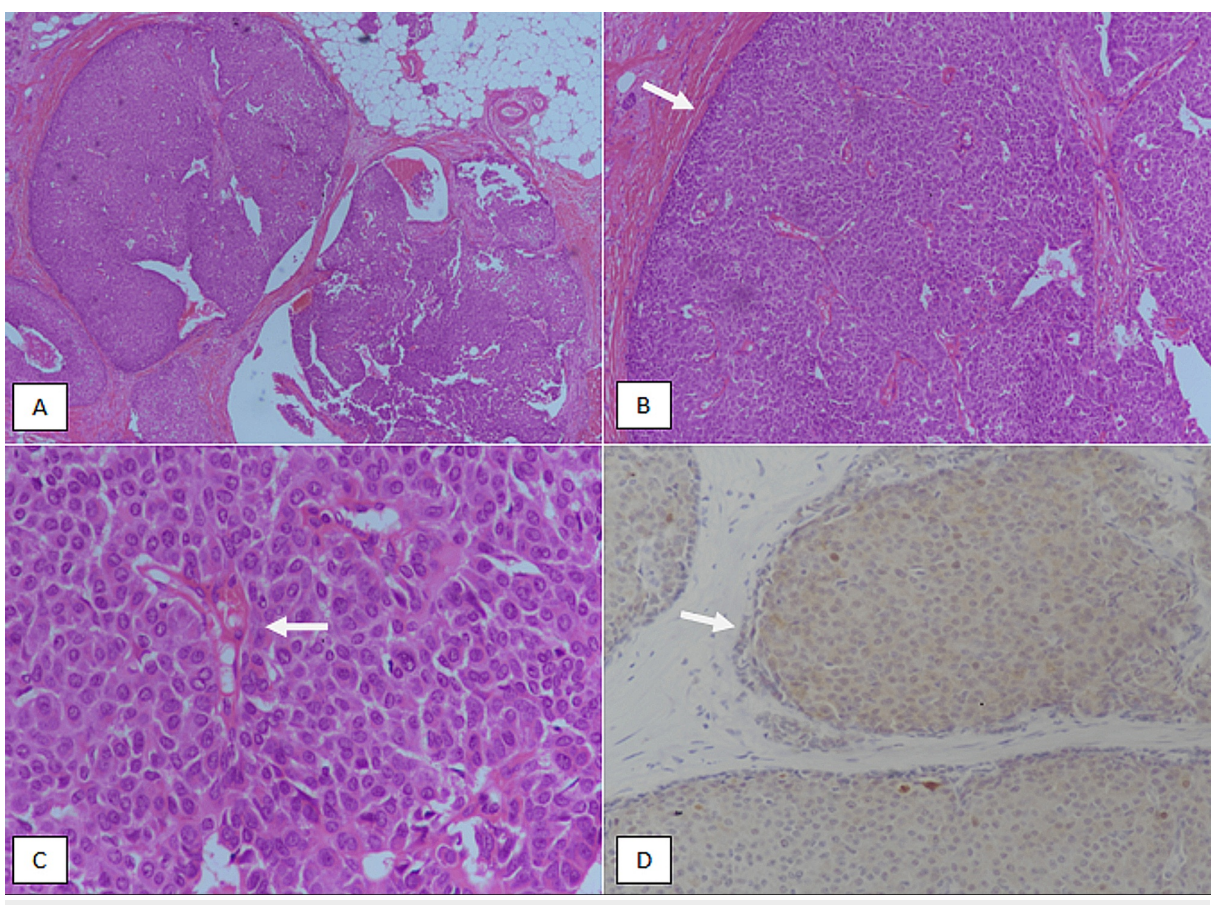

FIGURE 1: Solid papillary carcinoma in situ. (A): H \& E sections at 40X magnification showing discrete nodules of tumor. (B): 100X magnification showing circumscribed borders (arrow). (C): 200X magnification showing papillary cores (arrow) and low-grade nuclear atypia. (D): p63 immunostain showing lack of nuclear myoepithelial staining at the periphery of the tumor nodules.

$H \& E$, Hematoxylin and eosin 


\section{Cureus}

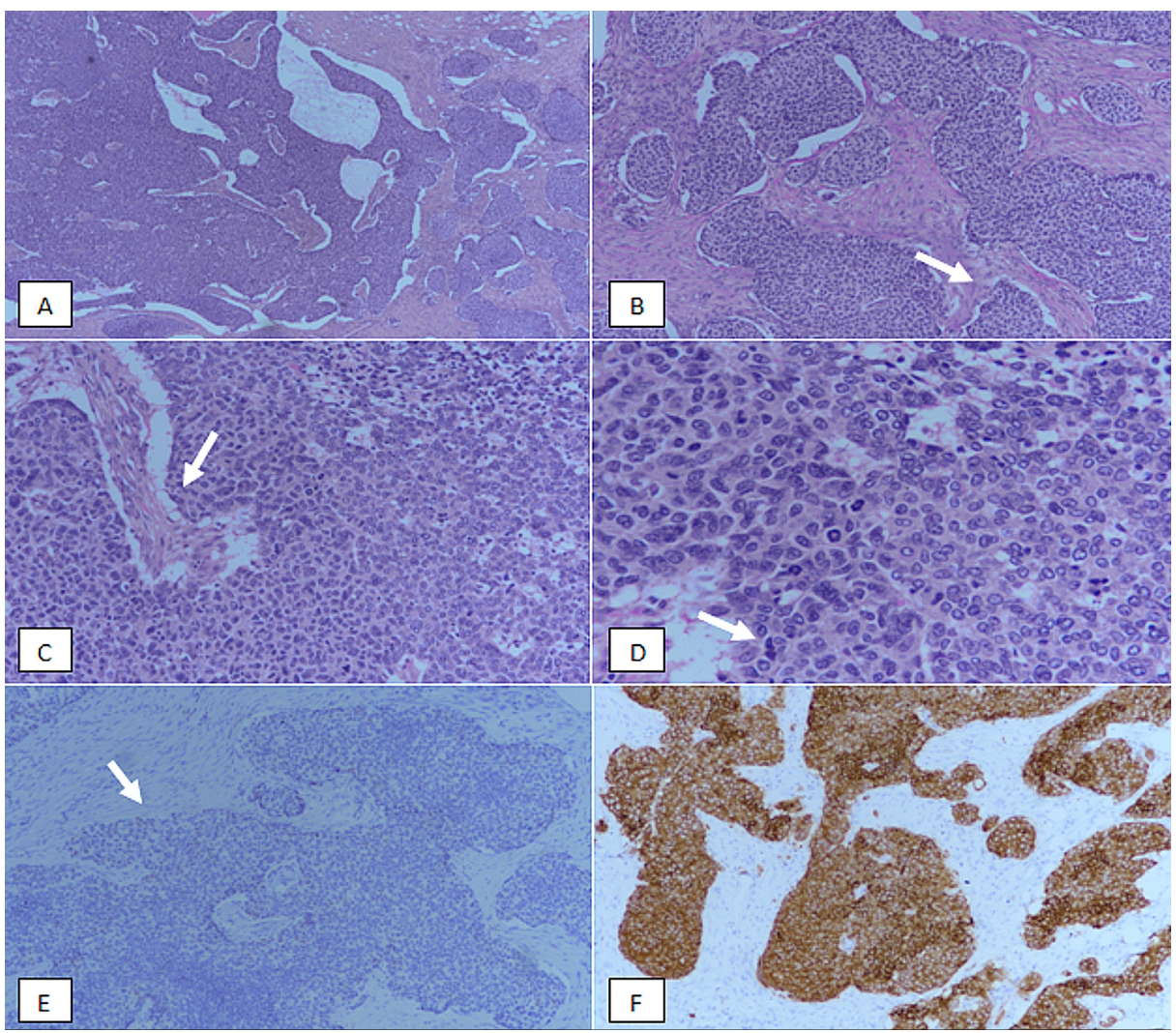

FIGURE 2: Solid papillary carcinoma invasive. (A): H \& E-stained sections at 40X magnification showing multiple nodules of a tumour. (B): 100X magnification showing irregular borders of the tumour (arrow). (C): 200X magnification revealing fibrovascular cores (arrow). (D): 400X magnification showing high-grade nuclear atypia with mitosis (arrow). (E): p63 immunostain showing lack of nuclear myoepithelial staining at the periphery of the tumour foci (arrow). (F): Synaptophysin immunostain revealing diffuse strong positivity in tumour cells.

$H$ \& E, Hematoxylin and eosin

Data analysis was performed using Statistical Package for Social Sciences (Version 26.0, IBM Inc., Armonk, USA). Chi-square, Fisher exact test and independent t-test were used to check the association. Survival analysis was performed by the Kaplan-Meier method. $p$-values $\leqslant 0.05$ were considered as significant.

\section{Results}

\section{Clinicopathological features of solid papillary carcinoma}

We included 39 cases of SPC in our study diagnosed during the study period. During the same timeline, 634 cases of IDC were reported and therefore included in the study for comparison. The mean age of the patients of SPC was $53.97 \pm 12.15$ years, and the mean tumour size was $3.42 \pm 1.87$. Majority of the specimens were lumpectomy (66.7\%), and most of the tumours were grade II (74.4\%). Axillary metastasis was noted in $15.4 \%$ of cases. $94.9 \%$ of cases of SPC were invasive. ER, PR, HER2/neu and synaptophysin positivity was seen in $84.6 \%, 87.2 \%, 10.3 \%$, and $59 \%$ respectively. Median follow-up time for SPC cases was 31 months. Recurrence was noted in $10.3 \%$ of cases with $94.9 \%$ survival rate. Detailed clinicopathological features of SPC cases under study are presented in Table 1 . 


\section{Cureus}

\begin{tabular}{|c|c|}
\hline \multicolumn{2}{|l|}{ Age groups } \\
\hline$\leq 50$ years & 19(48.7) \\
\hline$>50$ years & 20(51.3) \\
\hline \multicolumn{2}{|l|}{ Tumor size (cm) } \\
\hline Mean \pm SD & $3.42 \pm 1.87$ \\
\hline \multicolumn{2}{|l|}{ Tumor size groups } \\
\hline$<2 \mathrm{~cm}$ & $4(10.3)$ \\
\hline $2-5 \mathrm{~cm}$ & 29(74.4) \\
\hline$>5 \mathrm{~cm}$ & 6(15.4) \\
\hline Follow up (months) Mean \pm SD (median) & $30.66 \pm 10.49(31)$ \\
\hline \multicolumn{2}{|l|}{ Specimen type } \\
\hline Lumpectomy & 26(66.7) \\
\hline Simple mastectomy & $3(7.7)$ \\
\hline MRM & 10(25.6) \\
\hline \multicolumn{2}{|l|}{$\mathrm{N}$ stage } \\
\hline No & 33(84.6) \\
\hline N1 & $3(7.7)$ \\
\hline N2 & $3(7.7)$ \\
\hline N3 & $0(0)$ \\
\hline \multicolumn{2}{|l|}{ T stage } \\
\hline Tis & $2(5.1)$ \\
\hline T1 & $6(15.4)$ \\
\hline T2 & $26(66.7)$ \\
\hline T3 & $5(12.8)$ \\
\hline \multicolumn{2}{|l|}{ Grade } \\
\hline Grade-I & $7(17.9)$ \\
\hline Grade-II & $29(74.4)$ \\
\hline Grade-III & $3(7.7)$ \\
\hline \multicolumn{2}{|l|}{ Lymphovascular invasion } \\
\hline Present & $5(12.8)$ \\
\hline Absent & $34(87.2)$ \\
\hline \multicolumn{2}{|l|}{ Axillary metastasis } \\
\hline Present & $6(15.4)$ \\
\hline Absent & $33(84.6)$ \\
\hline \multicolumn{2}{|l|}{ ER } \\
\hline Positive & $33(84.6)$ \\
\hline Negative & 6(15.4) \\
\hline \multicolumn{2}{|l|}{ PR } \\
\hline Positive & $34(87.2)$ \\
\hline Negative & $5(12.8)$ \\
\hline
\end{tabular}




\section{Cureus}

HER2/neu

Positive

4(10.3)

Negative

35(89.7)

Synaptophysin

Positive

23(59)

Negative

16(41)

Type of SPC

SPC in situ

SPC invasive

37(94.9)

Recurrence

Yes

No

35(89.7)

Survival status

Alive

Expired

\section{TABLE 1: Clinicopathologic characteristics of Solid papillary carcinoma}

SD, standard deviation; MRM, modified radical mastectomy; T, tumour; N, nodal; Tis, tumor in situ; ER, estrogen receptor; PR, progesterone receptor; HER2/neu, human epidermal growth factor receptor-2; SPC, solid papillary carcinoma

\section{Comparison of solid papillary carcinoma with invasive ductal carcinoma}

Table 2 compares the clinicopathologic features of SPC with IDC. Cases with SPC were found significantly to have a lower grade (grade I + II), tumor (T) and nodal (N) stage compared to IDC. Moreover, frequency of hormonal receptor expression (ER and PR) was higher and frequency of HER2/neu expression was lower compared to IDC. However, no significant association was noted with respect to age, tumor size and lymphovascular invasion.

\begin{tabular}{|c|c|c|c|}
\hline Clinicopathologic characteristics & Solid Papillary carcinoma $(n=39)$ & Invasive ductal carcinoma $(n=634)$ & P-value \\
\hline \multicolumn{4}{|l|}{ Age (years) } \\
\hline Mean $\pm S D$ & $53.97 \pm 12.15$ & $51.95 \pm 12.15$ & $0.318^{\star \star \star}$ \\
\hline \multicolumn{4}{|l|}{ Age groups } \\
\hline$\leq 50$ years & 19(48.7) & $306(48.3)$ & \multirow{2}{*}{$0.956^{\star}$} \\
\hline$>50$ years & $20(51.3)$ & $328(51.7)$ & \\
\hline \multicolumn{4}{|l|}{ I umor size $(\mathrm{cm})$} \\
\hline Mean $\pm S D$ & $3.42 \pm 1.87$ & $3.61 \pm 1.48$ & $0.444^{\star \star \star}$ \\
\hline \multicolumn{4}{|l|}{$\mathrm{N}$ stage } \\
\hline No & $33(84.6)$ & $320(50.5)$ & \multirow{4}{*}{$<0.0001^{*}$} \\
\hline N1 & $3(7.7)$ & $130(20.5)$ & \\
\hline N2 & $3(7.7)$ & $85(13.4)$ & \\
\hline N3 & $0(0)$ & $99(15.6)$ & \\
\hline I stage & & & \\
\hline
\end{tabular}




\section{Cureus}

Tis

$\mathrm{T} 1$

T2

T3

Grade

Grade-I

Grade-II

Grade-III

Lymphovascular invasion

Present

Absent

Axillary metastasis

Present

$6(15.4)$

Absent

ER

Positive

Negative

PR

Positive

Negative

HER2/neu

Positive

Negative

2(5.1)

6(15.4)

26(66.7)

$5(12.8)$

$7(17.9)$

29(74.4)

$3(7.7)$

$5(12.8)$

$34(87.2)$

$33(84.6)$

6(15.4)

$34(87.2)$

$5(12.8)$

$4(10.3)$
$35(89.7)$
157(24.8)

477(75.2)

314(49.5)

320(50.5)

$399(62.9)$

235(37.1)

323(50.9)

311(49.1)

$<0.0001^{*}$

223(35.2)

$0.007^{\text {** }}$

$<0.0001^{\star \star}$

$0.09^{*}$

$<0.0001^{*}$

$0.006^{*}$

411(64.8)

$0.001^{*}$

TABLE 2: Comparison of clinicopathologic characteristics of solid papillary carcinoma with invasive ductal carcinoma of breast

${ }^{*}$ Chi-square test was applied, ${ }^{\star \star}$ Fisher Exact test was applied, ${ }^{\star \star \star}$ Independent t-test was applied.

SD, standard deviation; N, nodal; T, tumor; Tis, tumor in situ; ER, estrogen receptor; PR, progesterone receptor; HER2/neu, human epidermal growth factor receptor-2

Survival analysis of solid papillary carcinoma for tumour grade and size

We evaluated the survival status of SPC cases with respect to tumour grade and size using Kaplan-Meier curves; however, no significant difference in survival was seen with p-values (log-rank) of 0.645 and 0.628 , respectively as presented in Figures 3,4. 


\section{Cureus}

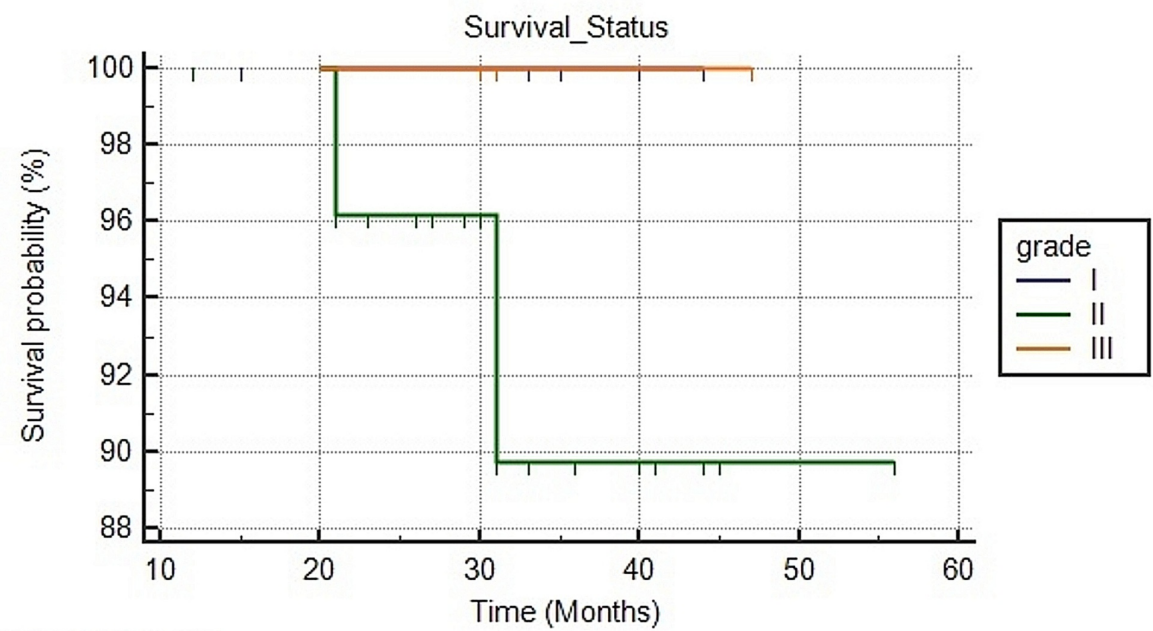

Number at risk

Group: I

\begin{tabular}{cccccc}
\multicolumn{1}{c}{7} & 6 & 6 & 1 & 0 & 0 \\
Group: II & & & \\
Group: III & 29 & 15 & 5 & 1 & 0 \\
3 & 3 & 2 & 1 & 0 & 0
\end{tabular}

FIGURE 3: Survival analysis using the Kaplan-Meier method for Solid papillary carcinoma with respect to tumor grade

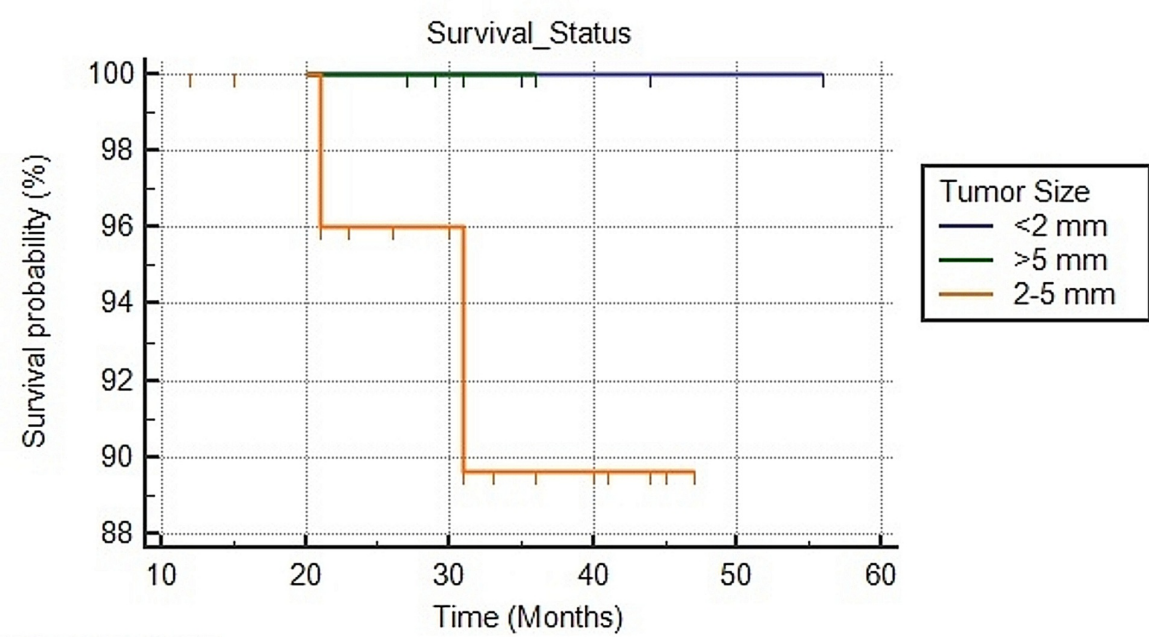

Number at risk

Group: $<2 \mathrm{~mm}$

$\begin{array}{cccccc}4 & 4 & 4 & 2 & 1 & 0 \\ \text { Group: }>\begin{array}{c}-5 \mathrm{~mm} \\ 7\end{array} & 6 & 4 & 0 & 0 & 0 \\ \text { Group: } 2-5 \mathrm{~mm} & & & & & \\ 28 & 25 & 15 & 5 & 0 & 0\end{array}$

FIGURE 4: Survival analysis using the Kaplan-Meier method for Solid papillary carcinoma with respect to tumor size

\section{Discussion}

In this study, we evaluated the clinicopathological features of SPC and compared with IDC diagnosed during the same study period. We found that SPC had overall better prognostic features than IDC as cases of SPC were found to have a lower grade, T-stage and N-stage than IDC. Moreover, the overall survival of SPC cases was over $90 \%$ with a low frequency of recurrence in our study. 
SPC is a distinct type of breast carcinoma that is staged as in situ carcinoma unless associated with invasive cancer or exhibit jagged infiltrative borders. A study conducted in Singapore evaluated the clinicopathologic characteristics of SPC. The authors concluded that invasive carcinoma associated with SPC was of small size and low grade [7]. In our study, more than $90 \%$ of SPC cases had an invasive component, and most were low grade (I and II).

SPC is considered an indolent variant of breast carcinoma. A study involving 20 cases of SPC did not reveal nodal metastasis in any case [8]. In our study, nodal metastasis was seen in $15.4 \%$ of cases, which is less compared to IDC (49.5\%). Another unique feature of SPC is a neuroendocrine differentiation. A study revealed that $45 \%$ of cases of SPC exhibited neuroendocrine differentiation [9]. In our series of cases, synaptophysin positivity was seen in 59\% cases. It is essential to differentiate SPC from IDC with neuroendocrine differentiation that lacks the typical nodular pattern and papillary cores characteristic of SPC.

We acknowledge a few limitations to our study, the most important of which is the small sample size. Second, the follow-up for cases of IDC was not available for comparison with SPC. Therefore, we recommend extensive prospective cohort studies to evaluate the prognosis of SPC in our population with a comparison of survival with IDC.

\section{Conclusions}

SPC is a distinct malignant papillary tumour of the breast with neuroendocrine differentiation and good overall survival. Pathologically, SPC possesses better prognostic parameters such as lower tumour grade and TN-stage than IDC. Histologically, SPC is characterized by discrete nodules of tumour cells with low-grade nuclei and circumscribed borders; however, it is of utmost importance to recognize the invasion as we found invasion in a high percentage of cases in our study. Like other papillary breast lesions, the role of myoepithelial stains is of crucial importance to differentiate SPC from papillary DCIS. In addition, synaptophysin immunostaining also have a diagnostic role in SPC as we found its positivity in a substantial number of cases of SPC. Therefore, it is essential to recognize histological and immunohistochemical characteristics of SPC, especially on trucut biopsy to prognostically stratify patients with breast cancer.

\section{Additional Information \\ Disclosures}

Human subjects: Consent was obtained by all participants in this study. Not needed issued approval Not needed. IRB not needed for retrospective studies. Animal subjects: All authors have confirmed that this study did not involve animal subjects or tissue. Conflicts of interest: In compliance with the ICMJE uniform disclosure form, all authors declare the following: Payment/services info: All authors have declared that no financial support was received from any organization for the submitted work. Financial relationships: All authors have declared that they have no financial relationships at present or within the previous three years with any organizations that might have an interest in the submitted work. Other relationships: All authors have declared that there are no other relationships or activities that could appear to have influenced the submitted work.

\section{References}

1. Hashmi AA, Edhi MM, Naqvi H, Khurshid A, Faridi N: Molecular subtypes of breast cancer in South Asian population by immunohistochemical profile and Her2neu gene amplification by FISH technique: association with other clinicopathologic parameters. Breast J. 2014, 20:578-585. 10.1111/tbj.12329

2. Hashmi AA, Naz S, Hashmi SK, et al.: Epidermal growth factor receptor (EGFR) overexpression in triplenegative breast cancer: association with clinicopathologic features and prognostic parameters. Surg Exp Pathol. 2019, 2:6. 10.1186/s42047-018-0029-0

3. Hashmi AA, Aijaz S, Khan SM, et al.: Prognostic parameters of luminal A and luminal B intrinsic breast cancer subtypes of Pakistani patients. World J Surg Oncol. 2018, 16:1. 10.1186/s12957-017-1299-9

4. Hashmi AA, Aijaz S, Mahboob R, et al.: Clinicopathologic features of invasive metaplastic and micropapillary breast carcinoma: comparison with invasive ductal carcinoma of breast. BMC Res Notes. 2018, 11:531. 10.1186/s13104-018-3623-z

5. Wei S: Papillary lesions of the breast: an update . Arch Pathol Lab Med. 2016, 140:628-643. 10.5858/arpa.2015-0092-RA

6. Saremian J, Rosa M: Solid papillary carcinoma of the breast: a pathologically and clinically distinct breast tumor. Arch Pathol Lab Med. 2012, 136:1308-11. 10.5858/arpa.2011-0227-RS

7. Tan BY, Thike AA, Ellis IO, Tan PH: Clinicopathologic characteristics of solid papillary carcinoma of the breast. Am J Surg Pathol. 2016, 40:1334-42. 10.1097/PAS.0000000000000702

8. Otsuki Y, Yamada M, Shimizu S, et al.: Solid-papillary carcinoma of the breast: clinicopathological study of 20 cases. Pathol Int. 2007 , 57:421-9. 10.1111/j.1440-1827.2007.02118.x

9. Guo S, Wang Y, Rohr J, Fan C, Li Q, Li X, Wang Z: Solid papillary carcinoma of the breast: a special entity needs to be distinguished from conventional invasive carcinoma avoiding over-treatment. Breast. 2016, 26:67-72. 10.1016/j.breast.2015.12.015 